\title{
Influência de aditivos químicos na produção de compósitos madeira- cimento de duas espécies tropicais da Amazônia
}

\author{
Influence of chemical additives in the production of wood-cement composites of \\ two tropical species from Amazonia
}

\author{
Vinicius Gomes de Castro', Rafael da Rosa AzambujaII, \\ Carlos Frederico Alice Parchen ${ }^{\mathrm{III}}$, Setsuo Iwakiri ${ }^{\mathrm{IV}}$
}

\begin{abstract}
Resumo
Este trabalho teve como objetivo avaliar o potencial de uso de três diferentes aditivos químicos $\left(\mathrm{CaCl}_{2}, \mathrm{MgCl}_{2}\right.$ e cal hidratada) na produção de compósitos madeira-cimento manufaturados com a mistura de partículas de madeira de costaneira de piãozinho (Eschweilera coriaceae (DC.) Mart) e matá-mata-branco (Eschweilera odora (poepp.) Miers) e cimento Portland tipo CP II-Z, na razão 1:2,75, pelo método de adensamento por vibrocompactação. Teste de compatibilidade entre a madeira e cimento mostrou que essas espécies são aptas para a produção de compósitos quando pré-tratadas com o uso de aditivos químicos na mistura. Com base nos resultados, pode-se concluir que: (I) As espécies do gênero Eschweilera estudadas são aptas para produção de compósitos madeira-cimento, sendo que compósitos produzidos com a madeira de piãozinho (Eschweilera coriaceae) obtiveram melhores resultados; (II) A adição de $\mathrm{CaCl}_{2}$ teve efeito positivo nas propriedades mecânicas dos compósitos de Eschweilera coriaceae; e (III) Não é recomendável o uso de cal hidratada como aditivo químico quando o método utilizado é o de vibrocompactação.
\end{abstract}

Palavras-chave: Aglomerante inorgânico; Vibrocompactação; Aceleradores de pega

\begin{abstract}
The aim of this work was to evaluate the potential use of three different chemical additives $\left(\mathrm{CaCl}_{2}, \mathrm{MgCl}_{2}\right.$ and hydrated lime) in the production of wood-cement composites made with particles from the slab wood of piãozinho (Eschweilera coriaceae (DC.) Mart), matá-mata branco (Eschweilera odora (poepp.) Miers) and Portland cement CP II-Z, with a wood:cement ratio of 1:2,75, by the vibro-compaction densification method. The compatibility test between wood and cement indicated that both species were suitable for the composites production when pre-treated and mixed with chemical additives. Main results: (I) The two species of the Eschweilera genus are suitable for wood-cement composite productions; Composites produced with piãozinho (Eschweilera coriaceae) particles presented better results (II) The use of $\mathrm{CaCl}_{2}$ had a positive effect on the mechanical properties of composites with Eschweilera coriaceae wood; and (III) The use of hydrated lime as chemical additives is not recommended when the method of densification by vibro-compaction is applied.
\end{abstract}

Keywords: Inorganic binder; Vibro-compaction; Chemical accelerators

\footnotetext{
Engenheiro Florestal, Dr., Professor do Departamento de Ciências Agronômicas e Florestais, Universidade Federal Rural do Semi-Árido, Av. Francisco Mota, 572, Costa e Silva, 59625-900, Mossoró (RN), Brasil. vinicius.castro@ufersa.edu.br (ORCID: 0000-0003-0174-0572)

II Engenheiro Florestal, Me., Doutorando do Davis College of Agriculture, Natural Resources and Design, West Virginia University, 4100 Agricultural Sciences Building, PO Box 6108, Morgantown (WV), USA. rafael.r.azambuja@gmail.com (ORCID: 0000-0002-1818-0287)

III Engenheiro Civil, Dr., Professor do Departamento de Construção Civil, Universidade Federal do Paraná, Campus IV, Jardim das Américas, 81531980, Curitiba (PR), Brasil. parchen@ufpr.br (ORCID: 0000-0001-5890-4772)

Iv Engenheiro Florestal, Dr., Professor Departamento de Engenharia e Tecnologia Florestal, Universidade Federal do Paraná, Av. Lothário Meissner, 632, Jardim Botânico, 80210-170, Curitiba (PR), Brasil. setsuo@ufpr.br (ORCID: 0000-0003-0390-3830)
} 


\section{Introdução}

O gênero Eschweilera é considerado o mais comum da região amazônica em ordem de abundância (STEEGE et al., 2006). A compatibilidade entre madeira de 27 espécies tropicais, dentre elas Eschweilera spp. e o cimento Portland já foi estudado por Vilela e Du Pasquier (1968). Os autores analisaram o grau de inibição da madeira, comparando as temperaturas máximas de hidratação da mistura madeira-cimento com a do cimento puro. A madeira de Eschweilera sp., conhecida no Brasil pelo nome popular de matá-mata, foi considerada incompatível, sendo que a mistura não atingiu uma temperatura máxima dentro das primeiras 24 horas.

Contudo, a inibição à pega do cimento devido à presença de madeira com substâncias inibitórias pode ser diminuída com a adição de aditivos químicos. O cloreto de cálcio $\left(\mathrm{CaCl}_{2}\right)$ é a substância mais utilizada como aditivo devido à disponibilidade no mercado, baixo custo e resultados comprovados por décadas de uso e estudo. $\mathrm{O} \mathrm{CaCl}_{2}$ não só altera a taxa de hidratação dos minerais do cimento, mas também reage com eles, influenciando as propriedades de resistência mecânica, composição química, área superficial, morfologia e porosidade dos produtos da hidratação (RAMACHANDRAN, 1995).

Entretanto, para madeiras com alto índices de inibição à pega do cimento, o efeito acelerador do $\mathrm{CaCl}_{2}$ pode não ser suficiente para sobrepor a incompatibilidade entre as substâncias. Semple e Evans (2002) estudaram o efeito de diversos aceleradores para eliminar o efeito inibidor dos taninos polifenólicos presentes no cerne da madeira de Acacia mangium e concluíram que outros tipos de cloreto, dentre eles o cloreto de magnésio $\left(\mathrm{MgCl}_{2}\right)$, são mais eficientes do que o cloreto de cálcio. Porém, o uso de cloretos vem sendo questionado em materiais voltados para construção civil, por induzir a corrosão de materiais metálicos. Para a produção de compósitos madeira-cimento, uma possível alternativa seria o hidróxido de cálcio $\left(\mathrm{Ca}(\mathrm{OH})_{2}\right)$ como aditivo químico.

Além da compatibilidade seletiva entre madeira e cimento, outro possível gargalo para o desenvolvimento deste produto é o processo de fabricação. Tradicionalmente compósitos madeira-cimento incorporam 2,75 a 3 partes de cimento para 1 parte de madeira e são consolidados em prensas hidráulicas de pratos para gerar painéis com massa específica em torno de $1,25 \mathrm{~g} / \mathrm{cm}^{3}$ (NCL INDUSTRIES, 2011). Contudo, este método de produção, chamado de BISON, apresenta algumas limitações. A necessidade do uso do equipamento hidráulico exige que os compósitos sejam produzidos em fábricas especializadas, aumentando os custos de produção e eliminando a possibilidade de manufatura do compósito no próprio local de utilização. Como alternativa, Parchen (2012) sugeriu a substituição da prensagem pelo adensamento por vibração, técnica já utilizada na produção de blocos de concretos. Com auxílio de um vibrador, de baixo custo e fácil operação, a mistura madeira-cimento passa a ser compactada pela eliminação de ar e acomodação das partículas.

O objetivo deste trabalho foi analisar o efeito de diferentes aditivos químicos nas propriedades físicas e mecânicas de compósitos madeira-cimento produzidos pelo método de adensamento por vibrocompactação utilizando partículas de duas espécies florestais tropicais, piãozinho (Eschweilera coriaceae (DC.) Mart) e matá-mata-branco (Eschweilera odora (poepp.) Miers).

\section{Materiais e métodos}

Foram utilizadas madeiras de costaneiras das espécies conhecidas vulgarmente na região de coleta como piãozinho (Eschweilera coriaceae) e matá-mata-branco (Eschweilera odora), obtidas a partir do desdobro de toras em uma serraria localizada no município de Manaus, estado do Amazonas. A massa específica básica das madeiras foi determinada pelo valor médio da relação entre a massa da madeira seca pelo volume da madeira saturada determinado através do método estereométrico, por imersão em água destilada de cinco corpos de prova de cada espécie. Em 
seguida, as costaneiras, previamente descascadas, foram transformadas em partículas por um picador de disco e, em seguida, reduzidas em moinho de martelo.

Para o teste de compatibilidade entre madeira e cimento, foram utilizadas partículas classificadas em peneiras passantes na malha de $0,84 \mathrm{~mm}$ de abertura e retidas na de $0,5 \mathrm{~mm}$.

Uma quantidade de 90,5 ml de água destilada foi misturada com 200 gramas de cimento Portland tipo CP II-Z e 15 gramas de madeira, conforme recomendações de Weatherwax e Tarkow (1964), que sugerem o uso de $2,7 \mathrm{ml}$ de água por grama de madeira seca e um adicional de $0,25 \mathrm{ml}$ de água por grama de cimento.

Em um recipiente isolado termicamente e hermeticamente fechado, foi realizada a leitura das temperaturas de mistura através de termopares tipo "J", conectados a um módulo condicionador de sinais analógicos acoplado a uma placa de conversão analógico-digital. As leituras de temperatura ocorreram a cada minuto durante 24 horas para três repetições de cada espécie.

O grau de compatibilidade foi determinado pelo método $T_{R}$, sugerido por Olorunnisola (2008), em que a inibição é indicada pela razão entre o tempo de pega da mistura (tempo necessário para a reação atingir sua temperatura máxima) pelo tempo da pega do cimento sem adição de madeira. As madeiras foram classificadas de acordo com o valor médio de $T_{R}$ como: compatíveis $(\leq 1,5)$, aceitáveis (valores entre 1,5 e 2,0$)$ e incompatíveis $(\geq 2,0)$.

$\mathrm{Na}$ manufatura dos compósitos, as costaneiras foram previamente submersas em água fria por um período de 7 dias, sendo que a água foi trocada diariamente para promover a retirada de extrativos solúveis em água fria, os quais poderiam inibir a pega do cimento. Após secas e moídas, as partículas foram classificadas em peneira automática e selecionadas as passantes em peneira com abertura de $2,38 \mathrm{~mm}$ e retidas em abertura de $1,41 \mathrm{~mm}$. As áreas superficiais média das partículas foram calculadas de acordo com a fórmula sugerida por Moslemi (1974).

As partículas foram misturadas manualmente com cimento tipo Portland CP II - Z na relação madeira:cimento de 1:2,75, baseado no peso seco da madeira. Foram adicionados $0,5 \mathrm{ml}$ de água para cada grama de cimento e uma quantidade extra de $0,3 \mathrm{ml}$ de água para cada grama de madeira seca.

Os aditivos químicos utilizados foram o cloreto de cálcio $\left(\mathrm{CaCl}_{2}\right)$ e cloreto de magnésio $\left(\mathrm{MgCl}_{2}\right)$, os quais foram adicionados à mistura, na quantidade de $3 \%$ em relação ao peso seco do cimento. O hidróxido de cálcio $\left(\mathrm{Ca}(\mathrm{OH})_{2}\right)$, ou cal hidratada, foi preparado através da hidratação de óxido de cálcio $(\mathrm{CaO})$, com grau de pureza de $80 \%$, em proporções que resultaram em uma solução com $21,3 \%$ de $\mathrm{CaO}$. A solução foi adicionada na mistura seguindo a relação 1:1 com o peso seco da madeira.

Os compósitos de madeira-cimento foram produzidos pelo processo de adensamento por vibrocompactação sugerido por Parchen (2012). Os tratamentos foram depositados em moldes prismáticos de painéis compensados, com dimensões de $42 \mathrm{~cm}$ de comprimento, $7 \mathrm{~cm}$ de largura e $1,5 \mathrm{~cm}$ de espessura, e submetidos à mesa vibratória com uma carga de impacto vertical equivalente a $220 \mathrm{~N}$ a $3.000 \mathrm{RPM}$ por 30 segundos (Figura 1). Com a vibração ocorreu a sedimentação do material e o molde então foi preenchido com mais mistura e novamente submetido à vibração. Este processo foi repetido até não haver mais espaço livre no molde, mesmo após o período de vibração. Além de corpos de prova com a adição de $\mathrm{CaCl}_{2}, \mathrm{MgCl}_{2} \mathrm{e}$ $\mathrm{Ca}(\mathrm{OH})_{2}$, também foram produzidos compósitos das duas espécies do gênero Eschweilera sem a mistura de aditivos químicos como testemunha.

A mistura foi mantida dentro do molde por 7 dias. Após esse período, o molde foi desmontado e os corpos de prova foram condicionados em um local coberto, à sombra, sem controle de umidade ou temperatura, por 21 dias para se completar os 28 dias necessários para a cura do cimento. Após a cura, os corpos de prova foram cortados com serra diamantada nas dimensões de $50 \mathrm{~mm}$ de largura e comprimento, para atender às normas europeias para os testes de absorção de água e inchamento em espessura (ECS EN 317, 1993) e ligação interna (ECS EN $319,1993)$, e nas dimensões de $50 \mathrm{~mm}$ de largura e $350 \mathrm{~mm}$ de comprimento, para realização do teste flexão estática (ECS EN 310, 1993) (Figura 2). 
Figura 1 - Mesa vibratória (a) e molde prismático produzidos com painéis compensados preenchidos com a mistura madeira-cimento após processo de adensamento (b).

Figure 1 - Vibration table (a) and prismatic mold made from plywood filled with wood-cement mixture after the densification process $(b)$.

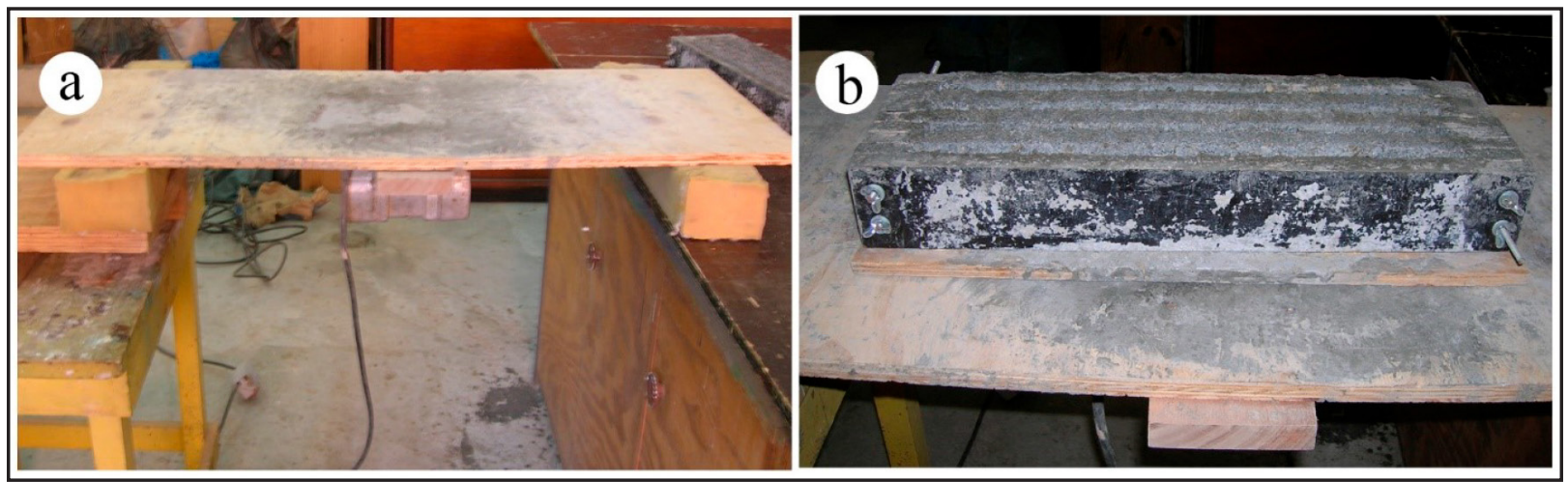

Fonte: Autores (2019)

Figura 2 - Avaliação das propriedades de ligação interna (a) e resistência à flexão estática (b) realizado nos compósitos madeira-cimento depois de dimensionados de acordo com a norma europeia.

Figure 2 - Evaluation of internal bonding (a) and static bending strength (b) properties of wood-cement composites after the cut according to the European standards.

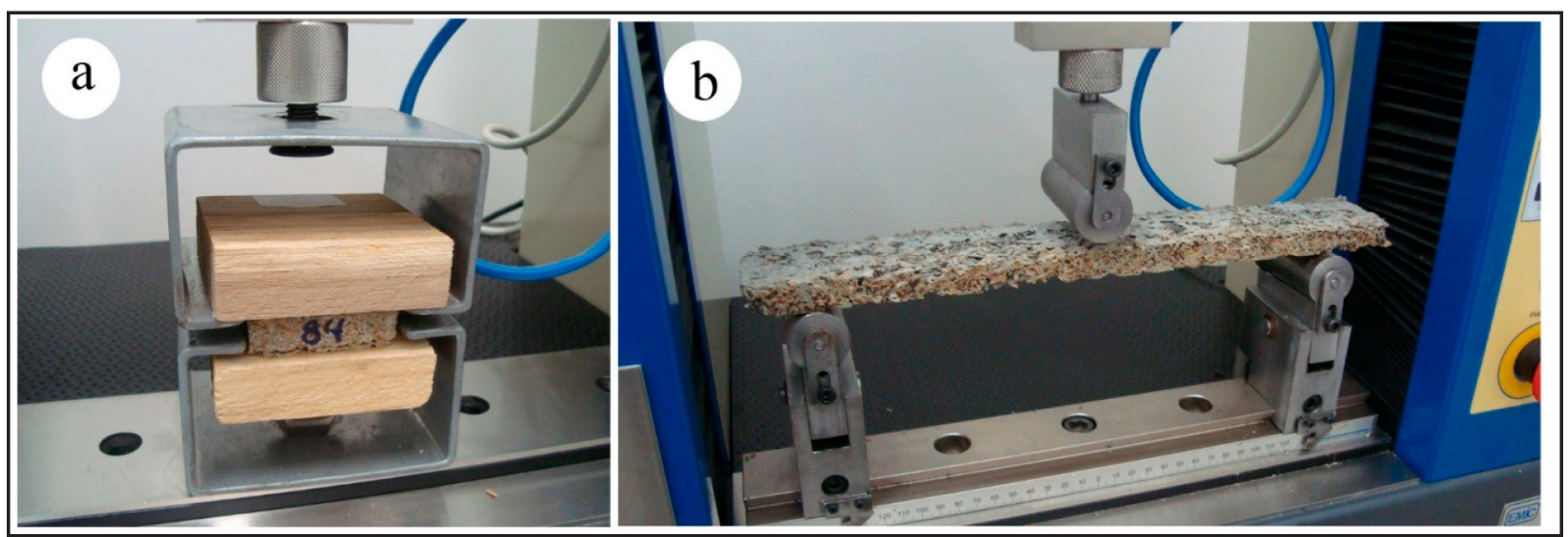

Fonte: Autores (2019)

Para verificar o efeito dos tratamentos sobre as propriedades dos compósitos, foi realizada uma análise de covariância considerando a massa específica covariável. Na verificação da existência de diferença estatística entre os tratamentos, o teste de Tukey foi aplicado, a 95\% de probabilidade

\section{Resultados e discussão}

A Tabela 1 apresenta os dados obtidos a partir das curvas da reação exotérmica de pega do cimento nas primeiras 24 horas, após, a mistura apenas com água (cimento puro) e com água mais partículas de madeira das duas espécies de Eschweilera. 


\section{Tabela 1 - Valores médios de temperatura e tempo de hidratação das misturas e graus de compatibilidade pelo método $T_{R}$.}

Table 1 - Average values for hydration temperature and time of mixtures and the compatibility degree by $\mathrm{T}_{\mathrm{R}}$ method.

\begin{tabular}{lcccc}
\hline & $\mathbf{T}_{\text {máx. }}\left({ }^{\circ} \mathbf{C}\right)$ & $\mathbf{t}$ (horas) & $\mathbf{T}_{\mathrm{R}}$ & Compatibilidade \\
\hline $\begin{array}{l}\text { Cimento puro } \\
\text { Eschweilera }\end{array}$ & 51,0 & 12,1 & - & - \\
$\begin{array}{l}\text { coriaceae } \\
\text { Eschweilera odora }\end{array}$ & 37,0 & 18,5 & 1,53 & Aceitável \\
\hline
\end{tabular}

Em que: $\mathrm{T}_{\text {máx. }}=$ temperatura máxima de hidratação em ${ }^{\circ} \mathrm{C} ; \mathrm{t}=$ tempo para se atingir a temperatura máxima em horas; $\mathrm{T}_{\mathrm{R}}=$ índice de compatibilidade entre madeira e cimento.

De acordo com o teste de compatibilidade $T_{R}$, a madeira das duas espécies estudadas apresentaram uma inibição aceitável à pega do cimento, ou seja, podem ser recomendadas para a manufatura de compósitos madeira-cimento, desde que se utilizem aditivos químicos como aceleradores de pega do cimento.

A Tabela 2 indica uma análise de variância fatorial na qual demonstra o efeito do aditivo químico, da espécie de madeira utilizada e a interação destes fatores na massa específica e nas propriedades mecânicas dos compósitos.

A massa específica final dos compósitos foi influenciada tanto pela espécie utilizada quanto pelo tipo de aditivo presente na mistura inicial. Não houve interação significativa entre os fatores avaliados para esta propriedade. Para a ligação interna, o efeito dos tratamentos foi significativo, sendo basicamente influenciado pelo uso ou não de aditivo na mistura. $\mathrm{O}$ efeito dos tratamentos também foi significativo para a resistência mecânica à flexão estática, sendo influenciado pela interação dos fatores aditivo e espécie.

A Figura 3 apresenta os valores médios obtidos para a massa específica e para as propriedades mecânicas dos compósitos madeira-cimento.

Compósitos produzidos com partículas de madeiras de Eschweilera coriaceae apresentaram valores médios para massa específica significativamente superiores aos produzidos com partículas de Eschweilera odora. No método de adensamento por vibrocompactação, a massa específica final do compósito dependente de dois fatores: o volume de material depositado nos moldes e a acomodação das partículas durante a vibração, uma vez que não há pressão na formação dos compósitos. Por sua vez, estas variáveis podem ser correlacionadas com a massa específica básica da madeira utilizada e a geometria das partículas. As espécies Eschweilera coriaceae e Eschweilera odora apresentaram massa específica básica estatisticamente semelhantes $\left(0,73\right.$ e $0,69 \mathrm{~g} / \mathrm{cm}^{3}$, respectivamente). Contudo, o valor médio da área superficial das partículas de Eschweilera coriaceae foi estatisticamente inferior $\left(84,24 \mathrm{~cm}^{2} / \mathrm{g}\right)$ ao das partículas de Eschweilera odora $\left(105,2 \mathrm{~cm}^{2} / \mathrm{g}\right)$.

O uso ou não de cloreto, tanto de cálcio quanto de magnésio, não afetou a densificação dos compósitos. Os compósitos produzidos com partículas de Eschweilera coriaceae e tratados com cal hidratada apresentaram uma média inferior ao dos demais tratamentos da mesma espécie. Essa menor massa específica pode ser explicada pela perda de material ocorrida durante o processo. $\mathrm{O}$ uso da solução de cal gerou uma mistura de menor viscosidade que escorreu pelas frestas dos moldes durante a vibração.

Quanto à propriedade de ligação interna dos compósitos sem uso de aditivos, observou-se um valor médio estatisticamente superior para os compósitos produzidos com partículas de Eschweilera coriaceae em relação aos produzidos com Eschweilera odora. Porém, a diferença entre os valores médios encontrados para esta propriedade deixou de ser estatisticamente significante quando aditivos químicos foram aplicados. 
Tabela 2 - Análise de variância fatorial em razão das propriedades físicas dos compósitos madeira-cimento.

Table 2 - Factorial analysis of variance as a function of physical properties of wood-cement composites.

\begin{tabular}{lcccccc}
\hline Variável & Fonte & GL & SQ & QM & F & P \\
\hline Massa & Aditivo & 3 & 0,25051 & 0,08350 & 18,26 & $0,000000^{*}$ \\
específica & Espécie & 1 & 0,94166 & 0,94166 & 205,89 & $0,000000^{*}$ \\
$\left(\mathbf{g} / \mathbf{c m}^{3}\right)$ & Interação AxB & 3 & 0,02946 & 0,00982 & 2,15 & 0,110284 \\
& Erro & 38 & 0,17380 & 0,00457 & & \\
\hline \multirow{2}{\text{Ligação}}{} & Aditivo & 3 & 0,264486 & 0,088162 & 12,61508 & $0,000007^{*}$ \\
Interna & Espécie & 1 & 0,019556 & 0,019556 & 2,79831 & 0,102368 \\
$(\mathbf{M P a})$ & Interação AxB & 3 & 0,053673 & 0,017891 & 2,56001 & 0,068838 \\
& Erro & 39 & 0,272556 & 0,006989 & & \\
\hline & Aditivo & 3 & 2,18417 & 0,728058 & 2,66911 & 0,057103 \\
MOR & Espécie & 1 & 0,97773 & 0,97773 & 3,58443 & 0,063894 \\
$(\mathbf{M P a})$ & Interação AxB & 3 & 7,47318 & 2,491059 & 9,13241 & $0,000059^{*}$ \\
& Erro & 52 & 14,18411 & 0,272771 & & \\
\hline & Aditivo & 3 & 1621805 & 540602 & 1,39336 & 0,25198 \\
MOE & Espécie & 1 & 4127 & 4127 & 0,01064 & 0,918256 \\
$(\mathbf{M P a})$ & Interação AxB & 3 & 3955313 & 1318438 & 3,39818 & $0,024456^{*}$ \\
& Erro & 52 & 20175149 & 387984 & & \\
\hline
\end{tabular}

Em que: $\mathrm{SQ}=$ soma de quadrado; $\mathrm{GL}=$ graus de liberdade; $\mathrm{QM}=$ quadrado médio; $\mathrm{F}$ = variável do teste $\mathrm{F} ; \mathrm{P}$ = valor de $\mathrm{P} ; \mathrm{MOR}=$ módulo de ruptura à flexão estática; $\mathrm{MOE}=$ módulo de elasticidade à flexão estática; $\mathrm{A}=$ aditivo; $\mathrm{B}=$ espécie; $\left(^{*}\right)$ Significativo a 95\% de probabilidade.

Para os compósitos de Eschweilera coriaceae não houve diferença estatística entre os valores médios de ligação interna quando se usou $\mathrm{CaCl}_{2}$. Já o uso de $\mathrm{MgCl}_{2}$ e cal hidratada geraram um efeito negativo nessa propriedade. No caso dos compósitos de Eschweilera odora, o uso dos dois cloretos não afetou a ligação interna em relação à testemunha, sendo que apenas o uso de cal hidratada resultou em valores estatisticamente inferiores.

O requisito mínimo para a propriedade de ligação interna sugerido para painéis de madeiracimento comerciais produzidos pelo processo BISON é de 0,4 MPa (NCL INDUSTRIES, 2011). O processo BISON é o método tradicional de produção de painéis madeira-cimento com uso de prensa de pratos. Porém, mesmo utilizando o método de vibração, os compósitos produzidos com partículas de Eschweilera coriaceae, sem aditivo e com adição de cloreto de cálcio, e compósitos produzidos com partículas de Eschweilera odora com adição de cloreto de cálcio apresentaram valores médios acima do requisito mínimo. Castro et al. (2015) avaliaram o uso de $\mathrm{CaCl}_{2}$ e $\mathrm{MgCl}_{2}$ na produção de compósitos madeira-cimento adensados por vibrocompactação com partículas de Eucalyptus saligna. Os autores concluíram que o uso dos cloretos melhorou a propriedade de ligação interna dos compósitos, sendo o uso de $\mathrm{CaCl}_{2}$ mais eficiente do que o uso de $\mathrm{MgCl}_{2}$. Embora não tenha sido observada a influência direta do gênero Eschweilera na ligação interna dos compósitos, os resultados divergentes da literatura indicam que a propriedade depende da espécie de madeira e sua interação com o tipo de aditivo químico utilizado. 
Figura 3 - Massa específica e propriedades mecânicas dos compósitos madeira-cimento produzidos com e sem adição de aceleradores de pega do cimento. Colunas acompanhadas de mesma letra indicam que não houve diferença estatística entre as médias.

Figure 3 - Specific gravity and mechanical properties of wood-cement composites made with and without addition of cement cure accelerators. Columns followed by the same letters indicate that there was no statistical difference between the average values.

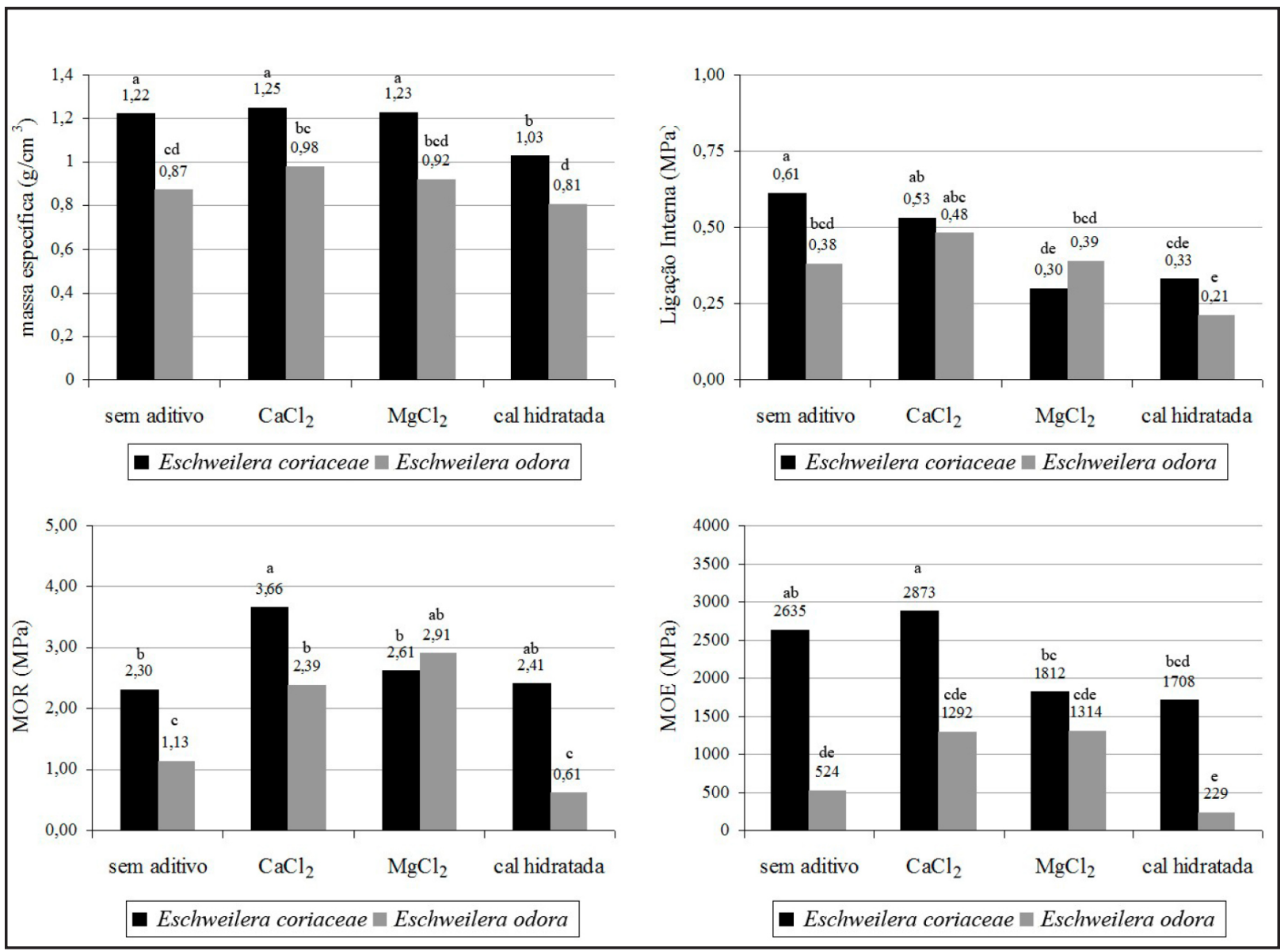

Fonte: Autores (2019)

Compósitos produzidos com Eschweilera coriaceae apresentaram valores médios de módulo de ruptura (MOR) estatisticamente superiores aos produzidos com Eschweilera odora, após ajuste considerando a massa específica dos compósitos como uma covariável. Este comportamento foi repetido com a adição de $\mathrm{CaCl}_{2}$ e cal hidratada, porém, o uso do aditivo $\mathrm{MgCl}_{2}$ resultou em valores médios estatisticamente semelhantes para os compósitos das duas espécies estudadas.

$\mathrm{O}$ uso de $\mathrm{CaCl}_{2}$ em compósitos com partículas Eschweilera coriaceae apresentou valores médios de MOR superiores à testemunha, enquanto que o uso dos demais aditivos não diferiu estatisticamente dos compósitos sem aditivos. Para os compósitos produzidos com Eschweilera odora, espécie de maior inibição à pega do cimento, os dois aditivos à base de cloreto apresentaram valores de MOR superiores à testemunha e iguais entre si, sendo que a adição de cal hidratada não afetou a propriedade.

O requisito mínimo do método BISON para a propriedade de MOR é estabelecido como $9 \mathrm{MPa}$ (NCL INDUSTRIES, 2011), valor que não foi atendido por nenhum dos tratamentos estudados. Entretanto, esta norma é voltada para painéis comerciais produzidos por processo 
de prensa de pratos, que resulta em partículas orientadas no sentido da espessura do painel. No processo de adensamento por vibrocompactação, não há orientação das partículas em nenhum sentido, o que resulta em um produto com características diferentes e de menor resistência mecânica. Parchen (2012) utilizou o processo de adensamento por vibrocompactação para a produção de compósitos madeira-cimento com partículas de Pinus spp. Embora o autor tenha utilizado uma relação madeira:cimento de 1:1, a massa específica dos compósitos foi de $0,8 \mathrm{~g} / \mathrm{cm}^{3}$, valor próximo aos compósitos produzidos com Eschweilera odora, permitindo a comparação das propriedades mecânicas. Os compósitos de Pinus spp. apresentaram um valor médio de MOR de $0,29 \mathrm{MPa}$, valor inferior aos encontrados para compósitos de Eschweilera, o que indica o potencial de uso da espécie.

Em relação ao efeito de aditivos no MOR à flexão estática, Wei, Zhou e Tomita (2000) observaram um comportamento semelhante aos dos compósitos de Eschweilera coriaceae ao estudarem o uso de $\mathrm{CaCl}_{2}$ e $\mathrm{MgCl}_{2}$ como aditivos químicos nas propriedades de compósitos produzidos com Betula albosinensis, espécie também considerada como de baixa inibição à pega do cimento $\left(\mathrm{T}_{\mathrm{R}}=1,13\right)$. $\mathrm{O}$ valor médio de MOR para compósitos sem aditivos foi de $3,53 \mathrm{MPa}$, as adições de $\mathrm{CaCl}_{2}$ e $\mathrm{MgCl}_{2}$ resultaram em melhoria da propriedade (13,26 e 6,59MPa, respectivamente).

Compósitos produzidos com partículas de Eschweilera coriaceae apresentaram valores médios do módulo de elasticidade (MOE) estatisticamente superiores a dos produzidos com Eschweilera odora. Contudo, o uso do aditivo $\mathrm{MgCl}_{2}$ resultou em valores estatisticamente semelhantes para a propriedade de MOE dos compósitos das duas diferentes espécies.

Para a propriedade de MOE, o uso de qualquer tipo de aditivo mostrou-se desnecessário, uma vez que não foi observada diferença estatística entre os tratamentos com ou sem aditivos. No caso específico dos compósitos produzidos com Eschweilera coriaceae, o uso de $\mathrm{MgCl}_{2}$ e de cal hidratada resultaram em valores médios estatisticamente inferiores à testemunha.

$\mathrm{O}$ requisito mínimo para a MOE sugerido para painéis madeira-cimento comerciais produzidos pelo método BISON é de $3.000 \mathrm{MPa}$ (NCL INDUSTRIES, 2011). Compósitos produzidos com partículas de Eschweilera coriaceae sem aditivo e com adição de $\mathrm{CaCl}_{2}$ apresentaram valores numericamente próximos ao requisito, mesmo sendo produzidos sem prensa. Castro et al. (2015) observaram que o uso de cloreto, de cálcio e de magnésio, teve efeito positivo nos valores de MOE de compósitos madeira-cimento produzidos com Eucalyptus saligna pelo método de vibrocompactação. O melhor resultado de MOE apresentado pelos autores foi para o tratamento com $\mathrm{MgCl}_{2}$ que apresentou um valor médio de $936 \mathrm{MPa}$, resultado inferior ao encontrados para compósitos produzidos com Eschweilera coriaceae. Os valores de MOE para compósitos com as madeiras amazônicas estudadas e sem aditivo também foram superiores ao maior valor médio (220 MPa) apresentado por Parchen (2012) para compósitos vibrocompactados utilizando partículas de diferentes granulometrias de galhos e troncos de Pinus spp.

$\mathrm{Na}$ Tabela 3 é apresentada a análise de variância entre as propriedades relacionadas à estabilidade dimensional e à espécie utilizada, o tipo de aditivo e a interação entre estes dois fatores.

Para todas as propriedades físicas relacionada à estabilidade dimensional houve influência da interação entre os fatores. Porém, a espécie utilizada para a produção dos compósitos não afetou as propriedades de absorção de água ou de inchamento em espessura após 24 horas de imersão. Sendo que o inchamento em espessura também não sofreu influência do tipo de aditivo usado, tanto após 2 ou 24 horas de imersão.

$\mathrm{Na}$ Figura 4 são apresentados os valores médios obtidos para as propriedades físicas referentes à estabilidade dimensional dos compósitos.

Após 2 e 24 horas de imersão em água, os compósitos produzidos com Eschweilera coriaceae absorveram menor quantidade de água que os produzidos com Eschweilera odora. As partículas de Eschweilera coriaceae apresentaram uma área superficial média menor que as partículas de Eschweilera odora, ou seja, a área de contato entre a madeira higroscópica e as moléculas de água foi menor nos compósitos de Eschweilera coriaceae. 


\section{Tabela 3 - Análise de variância fatorial em razão das propriedades de estabilidade dimensional dos compósitos madeira-cimento.}

Table 3 - Factorial analysis of variance as a function of dimensional stability of wood-cement composites.

\begin{tabular}{|c|c|c|c|c|c|c|}
\hline Variável & Fonte & GL & SQ & $\mathbf{Q M}$ & $\mathbf{F}$ & $\mathbf{P}$ \\
\hline \multirow{4}{*}{$\begin{array}{l}\text { Absorção de água } \\
\text { após } 2 \mathrm{~h}(\%)\end{array}$} & Aditivo & 3 & 118,573 & 39,524 & 17,835 & 0,000000 \\
\hline & Espécie & 1 & 39,178 & 39,178 & 17,678 & 0,000159 \\
\hline & $\begin{array}{c}\text { Interação } \\
\mathrm{AxB}\end{array}$ & 3 & 39,281 & 13,094 & \multirow[t]{2}{*}{5,908} & \multirow[t]{2}{*}{0,002123} \\
\hline & Erro & 37 & 81,998 & 2,216 & & \\
\hline \multirow{4}{*}{$\begin{array}{l}\text { Absorção de água } \\
\text { após } 24 h(\%)\end{array}$} & Aditivo & 3 & 162,5474 & 54,1825 & 11,4594 & 0,000019 \\
\hline & Espécie & 1 & 14,1414 & 14,1414 & 2,9909 & 0,092072 \\
\hline & $\begin{array}{c}\text { Interação } \\
\mathrm{AxB}\end{array}$ & 3 & 223,9050 & 74,6350 & \multirow[t]{2}{*}{15,7850} & \multirow[t]{2}{*}{$0,000001^{*}$} \\
\hline & Erro & 37 & 174,9441 & 4,7282 & & \\
\hline \multirow{4}{*}{$\begin{array}{l}\text { Inchamento em } \\
\text { espessura após } \\
2 \mathrm{~h}(\%)\end{array}$} & Aditivo & 1 & 0,022275 & 0,022275 & 3,07141 & 0,089258 \\
\hline & Espécie & 3 & 0,067151 & 0,022384 & 3,08634 & $0,041025^{\prime}$ \\
\hline & $\begin{array}{c}\text { Interação } \\
\mathrm{AxB}\end{array}$ & 3 & 0,171226 & 0,057075 & \multirow[t]{2}{*}{7,86977} & \multirow[t]{2}{*}{$0,000452^{\prime}$} \\
\hline & Erro & 32 & 0,232079 & 0,007252 & & \\
\hline \multirow{4}{*}{$\begin{array}{l}\text { Inchamento em } \\
\text { espessura após } \\
24 \mathrm{~h}(\%)\end{array}$} & Aditivo & 3 & 0,148325 & 0,049442 & 2,341365 & 0,090045 \\
\hline & Espécie & 1 & 0,030736 & 0,030736 & 1,455542 & 0,235733 \\
\hline & $\begin{array}{c}\text { Interação } \\
\mathrm{AxB}\end{array}$ & 3 & 0,631954 & 0,210651 & \multirow[t]{2}{*}{9,975606} & \multirow[t]{2}{*}{0,000068} \\
\hline & Erro & 35 & 0,739083 & 0,021117 & & \\
\hline
\end{tabular}

Em que: $\mathrm{SQ}$ = soma de quadrado; $\mathrm{GL}$ = graus de liberdade; $\mathrm{QM}=$ quadrado médio; $\mathrm{F}$ = variável do teste F; P = valor de P; A = aditivo; $\mathrm{B}$ = espécie; $\left(^{*}\right)$ Significativo a 95\% de probabilidade.

Em relação aos aditivos nas misturas, não houve efeito do uso de cloreto na propriedade de absorção de água após 2 e 24 horas de imersão para os compósitos produzidos com Eschweilera coriaceae. Observou-se um alto valor médio de absorção nos compósitos com adição de cal hidratada, que pode ter sido reflexo de uma maior exposição das partículas, devido à perda do material cimentício durante o processo de vibração. Para os compósitos produzidos com partículas de Eschweilera odora, o uso de $\mathrm{MgCl}_{2}$ resultou em corpos de prova com menor capacidade de absorção de água após 2 horas de imersão do que os tratamentos sem aditivo, mas após 24 horas de imersão, a diferença deixou de ser significativa.

Não houve diferença estatística significativa para o inchamento em espessura, após 2 ou 24 horas de imersão em água, entre os compósitos produzidos com partículas de Eschweilera coriaceae e Eschweilera odora. O uso de cloreto como aditivo químico também não apresentou diferença estatística para os valores de inchamento em espessura dos compósitos das duas espécies.

Os compósitos produzidos com partículas de Eschweilera coriaceae com adição de cal hidratada apresentaram valores médios de inchamento inferiores aos do tratamento com $\mathrm{MgCl}_{2}$. Uma possível explicação para este fenômeno foi a irregularidade e fragilidade da superfície dos corpos de prova produzidos com $\mathrm{Ca}(\mathrm{OH})_{2}$. Durante o processo de imersão dos corpos de prova em água houve degradação do material que resultou em um baixo valor na medição de espessura, mostrando não ser 
um indicativo de melhor estabilidade dimensional conforme se leva a crer pelos valores observados.

Figura 4 - Estabilidade dimensional dos compósitos madeira-cimento produzidos com e sem adição de aceleradores de pega do cimento. Colunas marcadas com a mesma letra indicam que não houve diferença estatística significante entre as médias.

Figure 4-Dimensional stability of wood-cement composites made with and without addition of cement cure accelerators. Columns followed by the same letters indicate that there was no statistical difference between the average values.

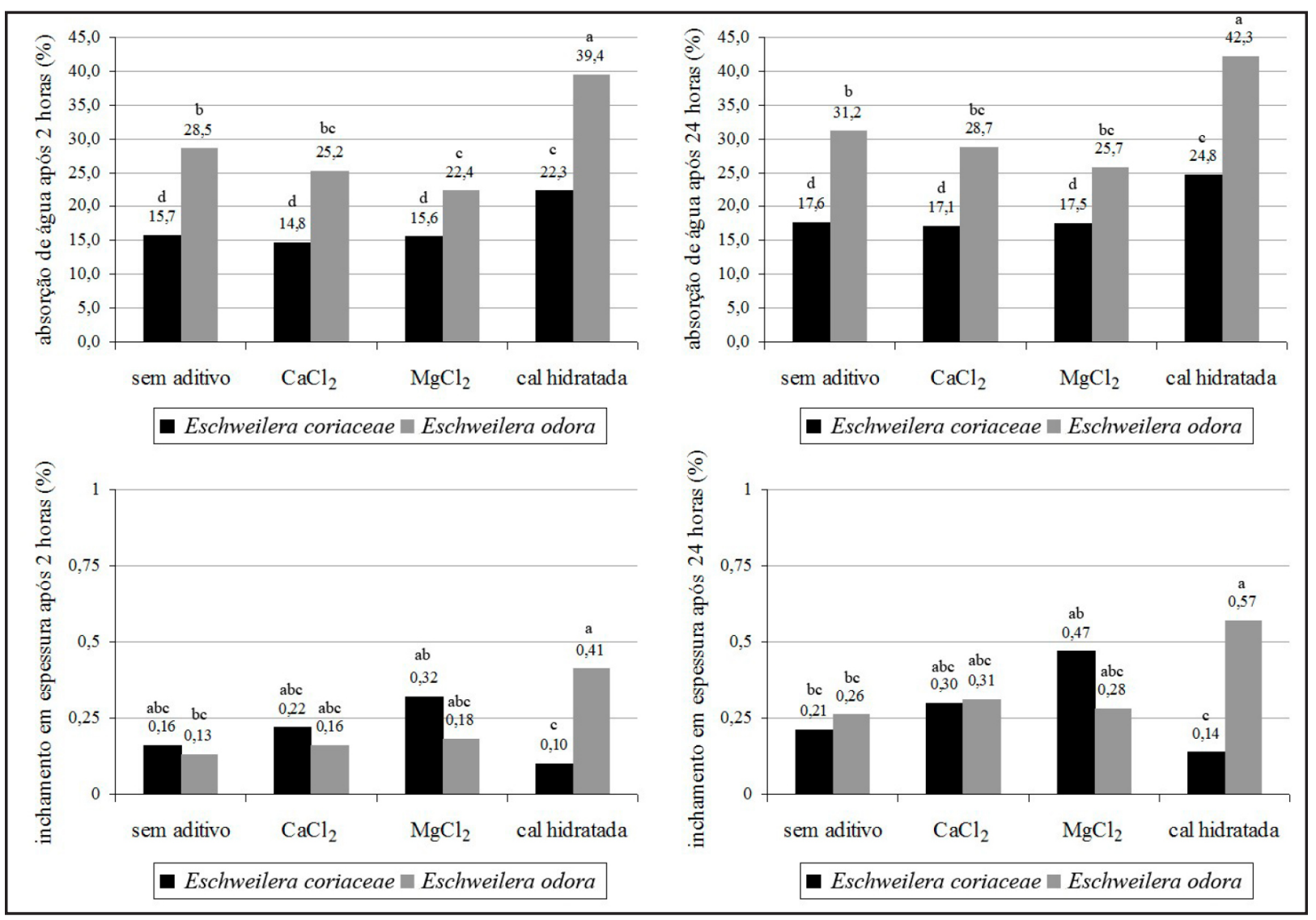

Fonte: Autores (2019)

Os resultados das propriedades físicas de compósitos de espécies de Eschweilera apresentaram alguns pontos divergentes ao comparado com os resultados de Castro et al. (2015), que avaliaram compósitos de Eucalyptus saligna e cimento produzidos pelo método de vibrocompactação. Os autores concluíram que o uso de cloreto de cálcio e de magnésio afetou a absorção de água tanto após 2 e 24 horas de imersão, porém, não houve diferença estatística nas propriedades de inchamento em espessura. Também não foi observada diferença significativa entre o uso de diferentes tipos de cloreto.

\section{Conclusão}

Com base nos resultados, pode-se concluir que:

- O uso das espécies Eschweilera coriaceae e Eschweilera odora é viável tecnicamente para a produção de compósitos madeira-cimento, sendo que o uso de Eschweilera coriaceae gera compósitos 
com melhores valores de ligação interna e resistência à flexão estática.

- Ao se produzir compósitos madeira-cimento com madeiras das duas espécies do gênero Eschweilera pelo método de vibrocompactação, não é recomendado o uso de aditivos químicos, uma vez que não foi observado efeito positivo significativo nas propriedades físicas e mecânicas do produto final. Sendo que a única exceção foi o aumento da resistência à ruptura em compósitos que utilizaram partículas de Eschweilera coriaceae com adição de $\mathrm{CaCl}_{2}$. Contudo, essa melhoria não foi suficiente para atender ao requisito mínimo desta propriedade para compósitos comerciais.

- O uso da cal hidratada como aditivo, de acordo com a metodologia proposta neste experimento, não é favorável à produção de compósitos madeira-cimento por vibrocompactação.

\section{Referências}

CASTRO, V. G. et al. Painéis cimento-madeira de Eucalyptus saligna com diferentes aditivos químicos e métodos de formação. Floresta, Curitiba, v. 25, n. 2, p. 349-360, 2015.

EUROPEAN COMMITTEE FOR STANDARDIZATION. EN 310. Wood-based panels - Determination of modulus of elasticity in bending and of bending strength. [S. l.], 1993.

EUROPEAN COMMITTEE FOR STANDARDIZATION. EN 317. Aglomerado de partículas de madeira e aglomerado de fibras de madeira - Determinação do inchaço na espessura depois da imersão em água. [S. l.], 1993.

EUROPEAN COMMITTEE FOR STANDARDIZATION. EN 319. Aglomerado de partículas de madeira e aglomerado de fibras de madeira - determinação da força da tensão perpendicular sobre a superfície plana da placa. [S. l.], 1993.

MOSLEMI, A. A. Particle geometry. In: MOSLEMI, A. A. Particleboard: Material. [S. l.]: Souther Illinois University, 1974. p. 49-67.

NCL INDUSTRIES. Bison panel - Cement bonded particle board. Abids, 2011. 24 p.

OLORUNNISOLA, A. O. Effects of pre-treatment of rattan (Laccosperma secundiflorum) on the hydration of Portland cement and the development of a new compatibility index. Cement and Concrete Composites, Amsterdam, v. 30, p. 37-43, 2008.

PARCHEN, C. F. A. Compósito madeira cimento de baixa massa específica produzido com compactação vibro dinâmica. 2012. Tese (Doutorado em Ciências Florestais) - Universidade Federal do Parará, Curitiba, 2012.

RAMACHANDRAN, V. S. Accelerators. In: RAMACHANDRAN, V. S. Concrete admixtures handbook - properties, science and technology. Noyes: New Jersey, 1995. p. 185-285.

SEMPLE, K. E.; EVANS, P. D. Screening inorganic additives for ameliorating the inhibition of hydration of Portland cement by the heartwood of Acacia mangium. In: EVANS, P. D. Wood-cement composites in the Asia-Pacific region. Cambera: ACIAR, 2002. p. 29-39. (ACIAR Proceedings, n. 107).

STEEGE, H. et al. Continental-scale patterns of canopy tree composition and function across Amazonia. Nature, London, v. 443, p. 444-447, 2006.

VILELA, E.; DU PASQUIER, B. Determinación del coeficiente de aptitud de un grupo de maderas para su posible aplicación em la fabricación de tableros de papilla de madera y cemento. Revista Forestal Venezolana, Mérida, v. 11, n. 16, p. 75-84, 1968.

WEATHERWAX, R. C.; TARKOW, H. Effect of wood on setting of portland cement. Forest Products Journal, Madison, v. 14, p. 567-570, 1964.

WEI, Y. M.; ZHOU, Y. G.; TOMITA, B. Study of hydration behavior of wood cement-based composite II: effect of chemical additives on the hydration characteristics and strengths of wood-cement composites. Journal of Wood Science, Tokyo, v. 46, p. 444-451, 2000. 